\title{
TRES ESPECIES NUEVAS DE STACHYS (LABIATAE) DE MEXICO'
}

\author{
Jerzy Rzedowski y Graciela Calderon de Rzedowskı \\ Instituto de Ecología, Centro Regional del Bajío \\ Apartado postal 386; 61600 Pátzcuaro, Mich. México
}

\section{RESUMEN}

Se describen como nuevas Stachys herrerana, del estado de Hidalgo, así como S. langmaniae y S. vulnerabilis, de Nuevo León. La primera se asemeja a S. venulosa Greene, conocida de Durango, mientras que las dos últimas parecen estar emparentadas entre sí, sin que pueda verse claramente su afinidad con otras especies.

\section{ABSTRACT}

Three Mexican species of Stachys are described as new: S. herrerana, from the state of Hidalgo, as well as $S$. langmaniae and $S$. vulnerabilis, from Nuevo León. $S$. herrerana seems to be similar to $S$. venulosa Greene, known from Durango, while $S$. langmaniae and $S$. vulnerabilis are perhaps related to each other, but their affinities with other species are obscure.

Durante una excursión realizada en 1984 a la Sierra de Pachuca los autores hallaron cerca de la población de El Guajolote una planta del género Stachys, que no pudo identificarse con ayuda de la literatura y de los herbarios que se tuvieron a la disposición. Se trata de un nuevo miembro de la flora del Valle de México, que se describe a continuación junto con otras dos especies de Nuevo León, colectadas por J. Hinton, depositadas en el herbario ENCB, y que se encuentran en el mismo caso.

\section{Stachys herrerana sp. $n$.}

Herba perennis usque ad $60 \mathrm{~cm}$ longa; caules ascendentes, hispidi et minute pubescentes; folia oblonga vel oblongo-lanceolata, (1)2-5 cm longa, (0.6) 0.8-1.8 cm lata, apice rotundata, margine grosse serrata, basi cuneata usque ad truncata, subtus conspicue nervata; verticillastri (2)4-6-flori, interrupti; calyx $\pm 6 \mathrm{~mm}$ longus, segmentis spinosis, corolla lilacina, ad labia maculis pallidioribus et obscurioribus, tubo $\pm 7 \mathrm{~mm}$ longo, infra medium ánnulato, labio superiore $5-6 \mathrm{~mm}$ longo, inferiore 7-8 $\mathrm{mm}$ longo et lato; stamina 2-3 $\mathrm{mm}$ e tubo exserta.

Planta herbácea perenne, hasta de $60 \mathrm{~cm}$ de largo, raíces fasciculadas, algo carnosas; tallos ascendentes, a menudo con una larga porción rastrera, llegando a arraigar en algunos nudos, poco o nada ramificados, entrenudos hasta de $12 \mathrm{~cm}$ de largo, híspidos con pelos de 0.5 a $1 \mathrm{~mm}$ de largo y además con tricomas más finos y cortos a menudo capitados; hojas con peciolos de (0)2 a $8(10) \mathrm{mm}$ de largo, por lo general más cortos hacia la parte superior de la planta, limbo oblongo a oblongo-lanceolado, de (1)2 a $5 \mathrm{~cm}$ de largo y de $(0.6) 0.8$ a $1.8 \mathrm{~cm}$ de

\footnotetext{
'Trabajo realizado con apoyo del Centro de Investigación y Desarrollo del Estado de Michoacán y del Consejo Nacional de Ciencia y Tecnología.
} 
ancho, ápice redondeado, borde toscamente aserrado, base cuneada a redondeada o a veces truncada 3-5-palmatinervado con la venación mucho más conspicua en el envés; inflorescencia interrupta, de 5 a $15 \mathrm{~cm}$ de largo, verticilastros de (2)4 a 6 flores, separándose con la edad hasta $7 \mathrm{~cm}$ entre sí, acompañados de dos hojas más o menos reducidas, pedicelos de $\pm 1 \mathrm{~mm}$ de largo; cáliz turbinado, de $\pm 6 \mathrm{~mm}$ de largo, densamente hispídulo, sus lóbulos de $1.5 \mathrm{a} 2 \mathrm{~mm}$ de largo, ápice agudo y con frecuencia provisto de una pequeña espina; corola de color lila con manchas más claras y más oscuras en los labios, tubo de $\pm 7 \mathrm{~mm}$ de largo, sin constricciones, anillado en la parte inferior, labio superior de 5 a $6 \mathrm{~mm}$ de largo, densamente hispídulo por fuera, labio inferior de 7 a $8 \mathrm{~mm}$ de largo y de ancho, hispídulo sobre las nervaduras; anteras de $\pm 1 \mathrm{~mm}$ de largo, negruzcas, sobresaliendo 2 a $3 \mathrm{~mm}$ del tubo de la corola, filamentos pubescentes, estilo glabro.

TIPO: México, Hidalgo, municipio de Epazoyucan, $1 \mathrm{~km}$ al $\mathrm{S}$ de El Guajolote, alt. $2800 \mathrm{~m}$, orilla de campos de cultivo en medio del bosque de Pinus y Juniperus, 25. VIII.1984, Rzedowski 38464 (ENCB, holotipo).

Especie hasta ahora conocida sólo de esta colecta.

S. herrerana pertenece al grupo VI de la clasificación provisional de Epling (1934) y al menos superficialmente parece asemejarse a S. venulosa Greene, descrita de Durango, de la cual no se han visto materiales auténticos. De acuerdo con la descripción, esa especie difiere en ser planta casi glabra, en las hojas de borde crenulado, en verticilastros con 6 a 12 flores y en los estambres sobresaliendo 3 a $3.5 \mathrm{~mm}$ del tubo de la corola.

El nombre de la especie se dedica a la memoria del distinguido biólogo mexicano Alfonso L. Herrera (1869-1942). Aunque el grueso de la voluminosa obra de Herrera se ubica fuera del ámbito de la botánica, cabe recordar su trabajo intitulado "El Valle de México considerado como provincia zoológica", que incluye el primer análisis fitogeográfico de dicha región.

\section{Stachys langmaniae sp. $n$.}

Herba perennis usque ad $70 \mathrm{~cm}$ alta; caules ad angulos retrorse hirsuti pilis basi pustulatis, $\pm 1 \mathrm{~mm}$ longis; folia deltoidea usque ad oblonga, (2)3-6 cm longa, $1-3 \mathrm{~cm}$ lata, apice rotundata, margine crenata, basi cordata vel truncata, subtus conspicue nervata; verticillastri 6flori, interrupti; caly x 5-6 mm longus, segmentis spinosis, corolla rosea, tubo 7-8 mm longo, infra medium annulato, labio superiore 2.5-3.5 mm longo, inferiore 6-8.5 mm longo, $\pm 6 \mathrm{~mm}$ lato; stamina $\pm 2 \mathrm{~mm}$ e tubo exserta.

Planta herbácea perenne, hasta de $70 \mathrm{~cm}$ de alto; tallos erectos o algo ascendentes, formando colonias densas, pero por lo general sin ramificarse, retrorsamente hirsutos con pelos moderadamente gruesos, de $\pm 1 \mathrm{~mm}$ de largo, pustulados en la base, la pubescencia total o casi totalmente concentrada en los ángulos, entrenudos hasta de $15 \mathrm{~cm}$ de largo; hojas con peciolos híspidos hasta de $2.5 \mathrm{~cm}$ de largo, pero en su mayoría mucho más cortos, limbo deltoideo a oblongo, variando en la inflorescencia a romboide, de (2)3-6 cm de largo, de 1 a $3 \mathrm{~cm}$ de ancho, ápice redondeado, borde crenado, base cordada a truncada al menos en la parte media $e$ inferior de la planta, 5-7-palmatinervado a partir de la base, con la venación conspicua en el envés, estrigoso en el haz con pelos moderadamente gruesos hasta de $1 \mathrm{~mm}$ de largo, en el 
envés la pubescencia está restringida a las nervaduras; inflorescencia ocupando a menudo $1 / 4$ a 1/3 de la longitud de la planta, interrupta y a veces ramificada, sus ejes densamente glanduloso-pubescentes, verticilastros por lo general con 6 flores, separándose con la edad hasta $4 \mathrm{~cm}$ entre sí, rodeados en su mayoría por brácteas linear-lanceoladas de $\pm 5 \mathrm{~mm}$ de largo, pedicelos de 0.5 a $1 \mathrm{~mm}$ de largo; cáliz turbinado a subcilíndrico (campanulado en fruto), de 5 a $6 \mathrm{~mm}$ de largo, densamente híspido con pelos de $\pm 1 \mathrm{~mm}$ de largo y además con pubescencia capitada más corta, sus lóbulos triangulares, de 2 a $2.5 \mathrm{~mm}$ de largo, acuminados en el ápice y terminando en una espina corta; corola rosada, tubo de 7 a $8 \mathrm{~mm}$ de largo, sin constricciones, anillado en la parte inferior, labio superior de 2.5 a $3.5 \mathrm{~mm}$ de largo, densamente hispídulo por fuera, el inferior de 6 a $8.5 \mathrm{~mm}$ de largo y $\pm 6 \mathrm{~mm}$ de ancho, hispídulo en las nervaduras; estambres exsertos $\pm 2 \mathrm{~mm}$ del tubo de la corola, filamentos pubescentes, anteras negruzcas, de $\pm 1 \mathrm{~mm}$ de largo, estilo glabro.

TIPO: México, Nuevo León, municipio Zaragoza, Encantada, alt. 2320 m, clearing in oak and pine forest, 17.VI.1979, Hinton \& al. 17545 (holotipo ENCB, isotipo IEB).

Material adicional examinado: México, Nuevo León, district Zaragoza, Picacho San Onofre, mixed forest, 25.VI.1978, Hinton \& al. 17419 (ENCB).

Los ejemplares arriba mencionados habían sido identificados como S. parvifolia Mart. \& Gal. vel aff. y como S. pringlei Greenm., pero S. langmaniae difiere de ambas especies en la pubescencia hirsuta de los tallos, que recuerda más bien los pelos de S. boraginoides Cham. \& Schl. Además, S. parvifolia tiene las flores más pequeñas y $S$. pringlei se caracteriza por los dientes del cáliz mucho más largos.

Es posible que $S$. langmaniae esté relacionada con $S$. vulnerabilis, otra especie de Nuevo León, que se describe en este artículo, e inicialmente se creyó que esta última sólo podría ser una forma de alta montaña de la primera. Sin embargo, $S$. vulnerabilis presenta otro tipo de pubescencia en sus tallos, tiene más largo el labio superior de la corola y menos salientes sus estambres, además de ser planta mucho más compacta.

El nombre de esta especie se dedica como homenaje a la Sra. Ida K. Langman, autora de "A selected guide to the literature on flowering plants of Mexico", obra de envergadura enciclopédica, de gran utilidad para todo tipo de estudiosos de las plantas de este país.

\section{Stachys vulnerabilis sp. $n$.}

Herba perennis usque ad $20 \mathrm{~cm}$ alta; caules retrorse hispidi et minute glandulosopubescentes; folia plerumque deltoidea, (1)2-3.5 cm longa, (0.3)1-2.3 cm lata, apice rotundata, margine crenata, basi subcordata vel truncata, subtus conspicue nervata; verticillastri 4-6-flori, interrupti; caly 6 6-7 mm longus, segmentis spinosis, corolla rosea, purpurea vel violacea, tubo 7-8 $\mathrm{mm}$ longo, infra medium annulato, labio superiore $3-5 \mathrm{~mm}$ longo, inferiore 7-8 $\mathrm{mm}$ longo et lato; stamina $\pm 1 \mathrm{~mm}$ e tubo exserta.

Planta herbácea perenne hasta de $20 \mathrm{~cm}$ de alto, a menudo formando colonias densas; tallos erectos o algo ascendentes, sin ramificarse, retrorsamente híspidos con pelos de 0.5 a 0.9 (2) $\mathrm{mm}$ de largo y además con pelos capitados hasta de $0.2 \mathrm{~mm}$ de longitud, los pelos largos concentrados a lo largo de los ángulos del tallo, entrenudos hasta de $4 \mathrm{~cm}$ de largo; hojas con 
peciolos hasta de $3 \mathrm{~cm}$ de largo, pero por lo general sólo de 0.5 a $1.5 \mathrm{~cm}$ de longitud, disminuyendo de tamaño hacia la parte superior de la planta, las de la inflorescencia a menudo casi sésiles, limbo por lo común deltoideo, variando a deltoideo-ovado o deltoideo-oblongo, en la inflorescencia elíptico o algo romboide, de (1)2 a $3.5 \mathrm{~cm}$ de largo y (0.3)1 a $2.3 \mathrm{~cm}$ de ancho, ápice redondeado, borde crenado, base subcordada a truncada en las hojas inferiores y de la parte media de la planta, 3-5-palmatinenado a partir de la base, con la venación muy conspicua en el envés, estriguloso en el haz, en el envés la pubescencia está restringida a las nervaduras; inflorescencia interrupta, hasta de $8(12) \mathrm{cm}$ de largo, verticilastros de 4 a 6 flores, separándose con la edad hasta $3 \mathrm{~cm}$ entre sí, rodeados por dos hojas reducidas, flores sobre pedicelos hasta de $2 \mathrm{~mm}$ de largo; cáliz turbinado a subcilíndrico (campanulado en la madurez), de 6 a $7 \mathrm{~mm}$ de largo, densamente hispídulo y con pelos capitados más cortos, sus lóbulos triangulares, de 2 a $2.5 \mathrm{~mm}$ de largo, agudos, terminando en una espina corta; corola de color rosa, morado o violeta (lavender, fide Hinton), tubo de 7 a $8 \mathrm{~mm}$ largo, sin constricciones, anillado en la parte inferior, labio superior de 3 a $5 \mathrm{~mm}$ de largo, densamente hispídulo por fuera, el inferior de 7 a $8 \mathrm{~mm}$ de largo y de ancho, hispídulo en las nervaduras; estambres exsertos $\pm 1 \mathrm{~mm}$ del tubo de la corola, filamentos pubescentes, anteras negruzcas, de $\pm 1 \mathrm{~mm}$ de largo, estilo glabro; mericarpios de $\pm 2 \mathrm{~mm}$ de largo, de color café.

TIPO: México, Nuevo León, Cerro El Potosí, cerca de la cima, municipio de Galeana, alt. $3460 \mathrm{~m}$, matorral de Pinus culminicola en bosque de Pinus hartwegii, en área perturbada por construcción de camino, 7.VII.1987, S. González 4029 (ENCB, holotipo; IEB, isotipo).

Material adicional examinado: México, Nuevo León, Cerro El Potosí, municipio de Galeana, alt. 3460 m, 7.VII.1987, A. García A., s.n. (ENCB, IEB); Cerro El Potosí, municipality of Galeana, $3800 \mathrm{~m}$, rocky summit, 20.VII.1969, Hinton \& al. 17201 (ENCB, IEB).

Ejemplares pertenecientes a este taxon, al parecer, han sido identificados como $S$. eriantha Benth., especie de altas montañas del centro de México, que difiere conspicuamente en sus hojas y flores mucho más pequeñas, con la corola sin anillo manifiesto en el tubo y sus labios sobresaliendo poco del cáliz, cuyos dientes son obtusos en el ápice.

Siguiendo la clave de Epling (op. cit.), S. vulnerabilis debería ubicarse cerca de S. exilis Epl. y S. globosa Epl. (del grupo V de su clasificación preliminar), pero no coincide con estas especies en su porte pequeño y los entrenudos cortos; además se diferencia de la primera en la forma de las hojas y en el tipo de pubescencia, y de la segunda en el tamaño de las flores $y$ en los verticilastros no globosos.

Quedan en duda, por consiguiente, las verdaderas afinidades de $S$. vulnerabilis, pero véase también la discusión correspondiente a $S$. langmaniae en este artículo.

El nombre $S$. vulnerabilis alude a la circunstancia de que la especie se encuentra aparentemente restringida en su distribución a la cima del Cerro Potosí, donde no es planta frecuente (Beaman \& Andresen, 1966). Esta zona, de dimensiones relativamente pequeñas, está siendo en la actualidad intensamente perturbada por las actividades ligadas a la operación de antenas de microondas así como por un intenso pastoreo y muchas de sus especies endémicas se encuentran en fuerte peligro de extinción. 


\section{AGRADECIMIENTOS}

Se dan las gracias a la Dra. Socorro González E. por haber puesto a nuestra disposición sus colectas de Stachys vulnerabilis. Se agradece asimismo a las autoridades del herbario ENCB por los materiales recibidos en préstamo.

\section{LITERATURA CITADA}

Beaman, J.H. \& J.W. Andresen. 1966. The vegetation, floristics and phytogeography of the summit of Cerro Potosi, Mexico. Amer. Midl. Natur. 75: 1-33.

Epling, C. 1934. Preliminary revision of American Stachys. Repert. Spec. Nov. Beih. 80: 1-75. 